\title{
Towards Mapping of Noise Impact
}

\section{Explaining ANIMA Efforts to Support New Approaches for Noise Impact Management Through Noise Management Toolset, Virtual Community Tool, and Dynamic Noise Maps}

\author{
Ferenc Marki $\mathbb{D}$, Peter Rucz $\mathbb{D}^{\text {, }}$, Nico van Oosten, Emir Ganić $\mathbb{D}^{\circ}$, \\ and Ingrid Legriffon $(\mathbb{D}$
}

\begin{abstract}
Noise impact management goes hand in hand with the capability to predict the noise impact on exposed communities. Three tools to that purpose are presented in this chapter: the Noise Management Toolset (NMT), the Demo Virtual Community Tool (VCT) and Dynamic Noise Mapping. The NMT is a web-based tool giving stakeholders the opportunity to evaluate scenarios through not only noise exposure, but also noise impact, by introducing annoyance related metrics like the awakening index, with an easy-to-use interface. The VCT is the underlying research tool exploring and testing new indicators and options that might be of relevance to target audiences, such as land use planning information about location dependent activities or window insulation. The third approach, Dynamic Noise Mapping, adds the important aspect of population movement to classical noise mapping approaches where temporal changes of noise maps are tracked and included in noise exposure evaluation.
\end{abstract}

Keywords Aircraft noise $\cdot$ Air traffic scenarios $\cdot$ Noise exposure $\cdot$ Annoyance $\cdot$ Perception oriented metrics $\cdot$ Land use planning $\cdot$ Human mobility patterns • National travel survey

F. Marki ( $\varangle)$ · P. Rucz

Budapest University of Technology and Economics, Budapest, Hungary

e-mail: marki@vik.bme.hu

P. Rucz

e-mail: rucz@hit.bme.hu

N. van Oosten

Anotec Engineering SL, Motril, Spain

e-mail: nico@anotecengineering.com

E. Ganić

University of Belgrade—Faculty of Transport and Traffic Engineering, Belgrade, Serbia

e-mail: e.ganic@sf.bg.ac.rs

\section{Legriffon}

ONERA, Université Paris Saclay, Chatillon, France

e-mail: ingrid.legriffon@onera.fr 


\section{Introduction}

When taking decisions with regard to land use planning, changes in air tracks etc., stakeholders need to rely on numbers. At present many tools are available that allow the user to generate noise exposure maps for airport scenarios. Although most of these tools have a rather sophisticated graphical user interface, their proper operation requires significant technical skills, usually only available at specialised consultants or at environmental departments of big airports. Due to the cost involved, this will normally limit the use of such tools to specific scenarios, required for compliance with legal requirements. It does thus usually not allow other interested stakeholders like land use planners, policymakers, airport staff, etc. to "play" with such tools to get a better understanding of the factors influencing airport noise management.

On the other hand, state-of-the-art tools usually only generate information on noise exposure. Although this is relevant for planning purposes, it falls short when airport noise issues need to be managed at a detailed level. As has been highlighted by the ANIMA project, an understanding of the reaction of people on interventions, aimed at reducing the noise impact, is required to maximise the benefits of such interventions.

Also, generating information on noise impact does not only imply knowledge of the noise sources in space and time, but also of the impacted population. Movement of people necessarily influences their exposure to noise and hence their perceived impact. Taking that varying parameter into account when estimating noise impact seems unavoidable, if it is to be done realistically.

In the following, three tools are presented. The above described shortcomings of current airport noise prediction models and mapping approaches have been addressed in ANIMA through the development of the Noise Management Toolset (NMT), the Virtual Community Tool (VCT) and Dynamic Noise Mapping. While the first one (NMT) offers a range of versions, going from a public version to a tool for aircraft noise experts, the second one (VCT) is a research tool elaborating, testing and validating new indicators, visualisations and options that can be implemented into the NMT if deemed of interest to stakeholders. The third approach, Dynamic Noise Mapping, adds the important aspect of population movement to classical noise mapping approaches.

\section{Noise Management Toolset}

\section{Objectives of the Tool}

The NMT has been developed with the aim to overcome the main shortcomings of existing airport noise tools, highlighted above. Therefore the main objectives of the tool are to: 
- Reduce the required technical skills so the tool can be used by a wider range of users

- Extend the scope from noise exposure to noise impact.

To further enhance the scope of the tool, the following additional forward-looking objective has been included:

- Allow for the inclusion of advanced aircraft/powerplant concepts (so as to study their noise impact, not only exposure)

\section{Working Principle}

In the European context airport noise models have to be compatible with the methodology described in ECAC Doc29 [1]. Therefore the NMT has been developed around the SONDEO model [2], which implements the latest version of this methodology. The design of SONDEO is such that it separates the complex noise calculations required to obtain the noise levels for each individual aircraft (the so-called "single events"), from the generation of the map that reflects the noise exposure of the full fleet operation, representative for a certain scenario. After this, impact related features can be calculated. Figure 1 provides a schematic representation of the workflow of the NMT.

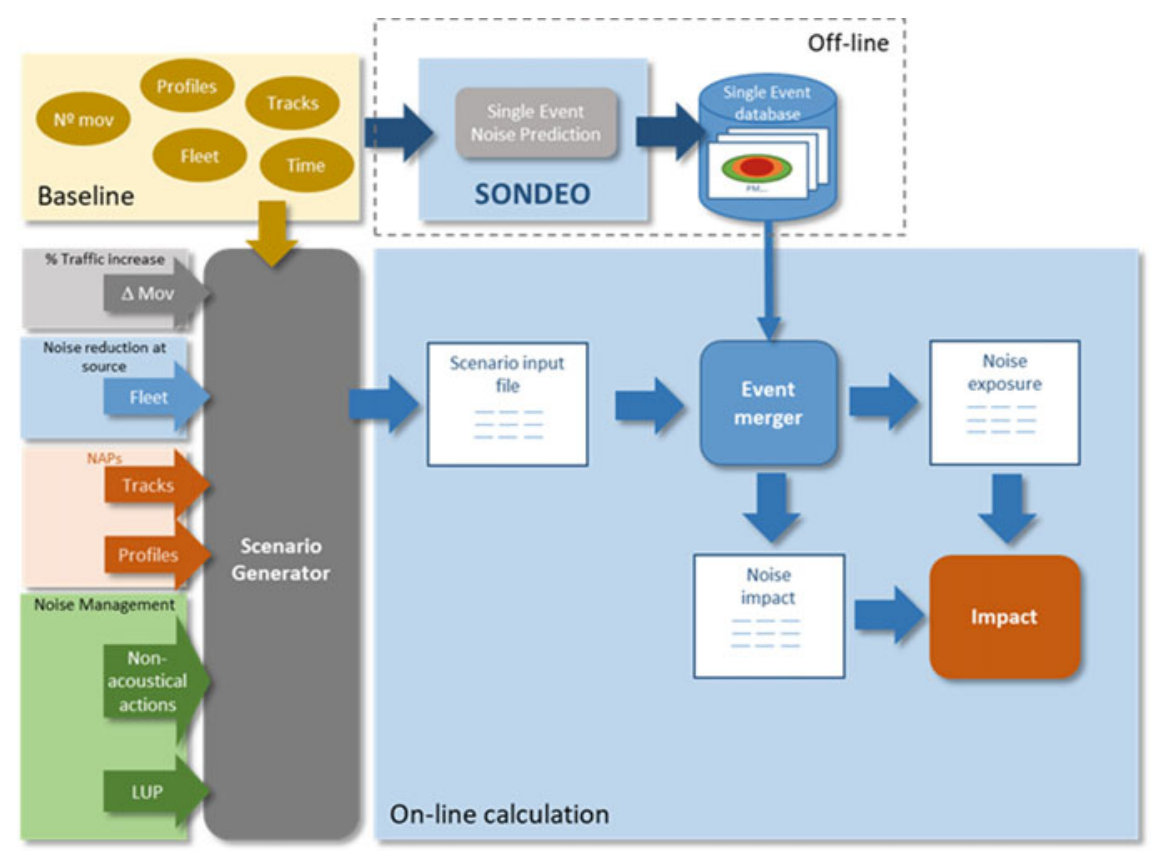

Fig. 1 Workflow of the noise management toolset 
The first step (generation of the single events database) inevitably requires in-depth technical knowledge of airport noise modeling. To avoid exposing the end-user to this part, the single-event database will be generated "off-line" by specialists (here called the NMT Administrator). This database will contain the individual noise footprint of each combination of aircraft type, flight track, flight profile, etc. that may be considered later on in the scenarios. With the support of additional simulation tools, the single events for any not yet existing aircraft can also be generated here. It is noted that the single event database will need to be generated only once.

The resulting single event database will be available at the start of the on-line user experience. The user can build his own scenarios by selecting the relevant combinations of single events and in this way generate the full set of operations for which the noise results shall be calculated. Apart from the standard noise exposure metrics, relevant noise impact related metrics are also calculated.

The results of the calculations are graphically presented and the user can select which results to display and can compare the results of various scenarios.

\section{Airports}

A so-called Public Toolset (PT) is available for the general public. The PT contains a virtual airport, with the aim to illustrate the basic concepts of airport noise mapping, explained in the ANIMA Best Practice Portal. To this end a set of traffic scenarios has been included, which the user can visualise and for which relevant information on the essential components (aircraft operations, tracks, noise contours, populated areas, etc.) can be displayed.

Apart from the PT, limited to the illustration of basic concepts, a premium version (the Noise Management Toolset or NMT) has been developed that addresses the needs of users such as land use planners, policymakers, airport staff, universities, etc. These users will want to be able to generate their own scenarios at a real airport, e.g. to design an intervention and assess its effect on the noise exposure and impact. Registration is required to obtain access to this advanced version of the NMT. As a first step, an authorised person (here called the Airport Owner) shall request inclusion of an airport in the NMT database, following the procedure described on the NMT website. This person shall have the permissions required to publish the information relevant for the noise calculations at that airport. The Airport Owner shall provide a dataset with which the NMT Administrator can generate the single event database and configure the system for inclusion of the new airport. This dataset will include information on the runway(s), standard flight routes (tracks), aircraft fleet, populated areas, etc. Once this database is ready, the airport will be available in the NMT and the Airport Owner can invite additional users to register for the new airport. Each NMT user will have access to all functionalities of the NMT for the virtual airport and the airport(s) (s)he has been assigned to. 


\section{Building of Scenarios}

The basic structure for the calculations to be done with the NMT is the Scenario. A Scenario represents a certain noise situation at an airport and as such consists of a specific combination of the single events available in the database. Each single event is defined by a unique combination of:

- Aircraft type

- Type of operation: Arrival or Departure

- Vertical flight procedure ("Profile")

- Distance flown (indicator for the weight at take-off)

- Runway

- Track

To define a Scenario the user should provide the following information for each single event (see Fig. 2):

- Number of operations

- Time of these operations (exact local time or period (Day, Evening, Night))

The NMT provides several options to create a new scenario. As a first option a scenario can be generated from scratch. In this case the user has to provide all the information on the aircraft operations. This can be done by manually filling in a table like the one presented in Fig. 2, or, more conveniently, by uploading a file containing the airport flight plan (similar to the time table usually managed at airports, i.e. providing the time of each individual operation) or a so-called operations file (resembling the table shown in Fig. 2, grouping the operations by period of the day, thus losing the time information of the individual operations). Templates for both are available on the NMT website. Usually the user will need to create the first scenario for an airport in one of these manners. However, once this is done, it is generally more convenient to clone the existing scenario and then change only those operations required to define the new scenario. Several smart features are available to assist the user in defining the new scenario. It is possible to move a certain amount of operations from one track to another, for all aircraft and time periods, or for specific

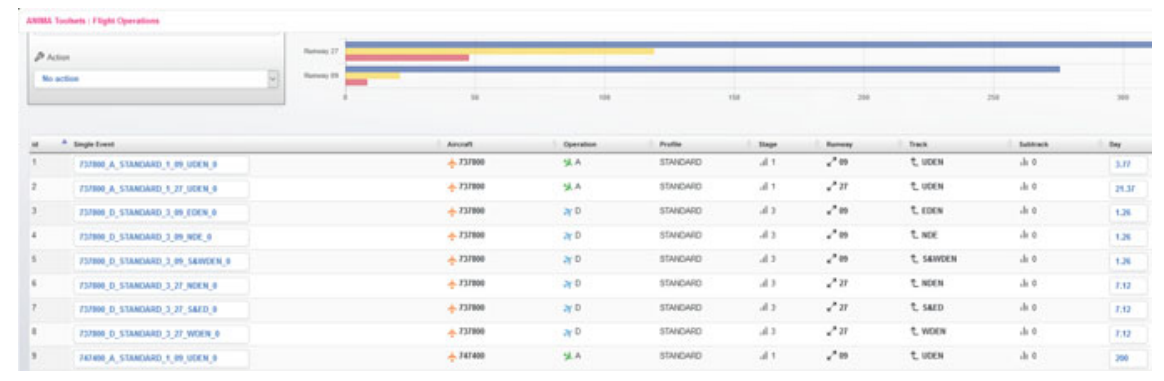

Fig. 2 Example of building a scenario, based on single events 
aircraft types and/or time periods individually. Similarly, it is possible to increase the number of operations by a certain percentage to easily simulate traffic growth.

Once a scenario has been created by one of the procedures explained above, the noise calculation is invoked, which will generate the noise exposure and impact maps.

\section{Visualisation of Results}

Once a scenario has been calculated the results can be visualised. The user can select the noise/impact contours that should be shown on the map. Information on track usage, specific populated areas, etc. can also be presented.

To assess the effect of the intervention represented by a scenario, the results (noise exposure and impact contours) of that scenario can be compared with those from another scenario. A maximum of 4 scenarios can be compared at the same time.

The reader is now encouraged to get a taste about the capability of the tool by visiting:

https://anima-project.eu/noise-platform/noise-management-toolset and by following the path along Noise Toolset to The Public Noise Toolset.

\section{Future Work}

Although the currently available NMT addresses the most relevant needs of the targeted stakeholders, further development of the tool is envisaged, to cater for additional functionalities that are considered of added value to the users.

Within ANIMA a more comprehensive desktop toolset has been developed for use by aircraft noise experts. Some of the functionalities implemented in this tool may be migrated to the web-based NMT. As a first additional feature, it is envisaged that the web-based NMT will be extended with the calculation of emissions (CO2 and NOx). In this manner the user will be able to obtain in a single execution both the noise and the emissions corresponding to a scenario. This will allow for the determination of the interdependencies between both environmental aspects and provide the user with means to perform trade-off studies.

As the title of the present chapter indicates, the NMT has been conceived with the objective to go beyond the mapping of noise exposure, by including aspects of noise impact. As this is a relatively new field of research, it is envisaged that new knowledge will be generated in the coming years. The Virtual Community Tool (VCT) described hereafter is the vehicle that will test the applicability of new insights in a representative airport environment. Once validated with the VCT, the new findings will be leveraged to the NMT. The web-based approach allows for an instantaneous upgrade of the NMT, allowing its users access to state-of-the-art knowledge on airport noise impact and its management. 


\section{Virtual Community Tool}

\section{Aim of the Tool}

As it was seen in former chapters, noise causes annoyance, but its amount cannot be clearly related to noise levels. Decision makers have a hard time trying to protect people living around airports: on the one hand they must use objective, measurable quantities, but on the other hand they should use something, which reflects people's subjective reactions to noise. Until now, separate daily, evening, and night-time noise levels, or their combination $\left(\mathrm{L}_{\mathrm{DEN}}\right)$ are calculated in most cases. But unfortunately these level metrics are normally computed for longer periods only, i.e., a month, the busiest six months, or a year, thus blurring the annoying effect of some worse days or even some hours of the day. Nevertheless, $\mathrm{L}_{\mathrm{DEN}}$ could already be seen as something that is at least a bit perception oriented, because it gives a penalty weighting for the evening and night hours, taking the more adverse effect of noise during these hours into account. Unfortunately it is, by far, not "human friendly" enough, as it absolutely does not consider the nature of aircraft noise being a series of individual events in contrast to much more continuous noises. $\mathrm{L}_{\mathrm{DEN}}$ can neither take critical hours into consideration, such as trying to get some rest outside after work, the time when people try to fall asleep, or when they are in a light-sleep phase soon before getting up; nor can it tell too much about sleep disturbance by the noise. (Similar findings are described in [3], which thus recommends that "supplementary Single Event metrics are routinely published by airports to better reflect the way in which noise is experienced on the ground".) Besides the metrics utilised, another issue with the current noise computation approach is the computational cost. The strategies applied currently are time consuming thus it is too costly to analyse various scenarios, like the rearrangement of flight hours or the increase of flight traffic in the future, or the renewal of the fleets, etc.

The introduction of new metrics to use as an evaluation tool on the harmful effect of air traffic is not just a difficult scientific task, it is also a hard decision, because there does not exist a universal, undisputed metric. Each of the metrics utilised nowadays emphasises one certain effect of noise, while suppressing the others. Instead of trying to invent new formulas of combined metrics, in ANIMA we try to move the current, mainly level-oriented decision approach into a direction, where more factors are considered. Therefore a tool has been developed, which is able to compute several metrics, each being strongly related to annoyance. A strong emphasis has also been set on the ability of the tool to easily change scenarios, i.e., to quickly analyse various possibilities. We don't know yet whether such a tool could be accepted by decision makers therefore we call our tool the "Demo Virtual Community Tool" (referred as "demo VCT") — as it demonstrates a new approach on evaluating aircraft noise effects.

When comparing it to the NMT, it is developed in a computer language, which allows very fast program development, so we can quickly implement whatever we think it could be useful, but it cannot correctly support user right management. 
Therefore it is good as an experimentation tool to find useful features that could be later on implemented in a more commercial-like software, like the NMT.

Please note that all examples presented here serve only to demonstrate the capability and the potential of the tool. Although you'll see computed metrics around Budapest Airport, the applied schedules are fictional, so it is strongly emphasised that the reader shall not draw any conclusions on the situation around BUD airport.

\section{Working Principle}

Figure 3 displays the overall workflow of the tool. There are two main inputs that are required for the computations performed by the Virtual Community Tool. First, an airport database must be available. This database contains the ground acoustical data (i.e. acoustical footprints) of all possible flight operations of a single airport. The dataset is sampled over a geographical grid that covers a given area in the vicinity of the given airport. The airport database may also be supplemented by additional regional information, containing a demographic map of the population density, an insulation map of the buildings in the area and further auxiliary data. The second input is called a flight schedule and it contains a list of actual operations performed in a given frame of time. A typical schedule may contain all operations of a week or a fortnight, whereas short schedules of a single day, or long schedules containing the traffic over a whole year are also handled by the tool. Once a schedule is available, all of its flights are matched to the airport database in order to establish the connection of the actual operation with the corresponding acoustical footprint.

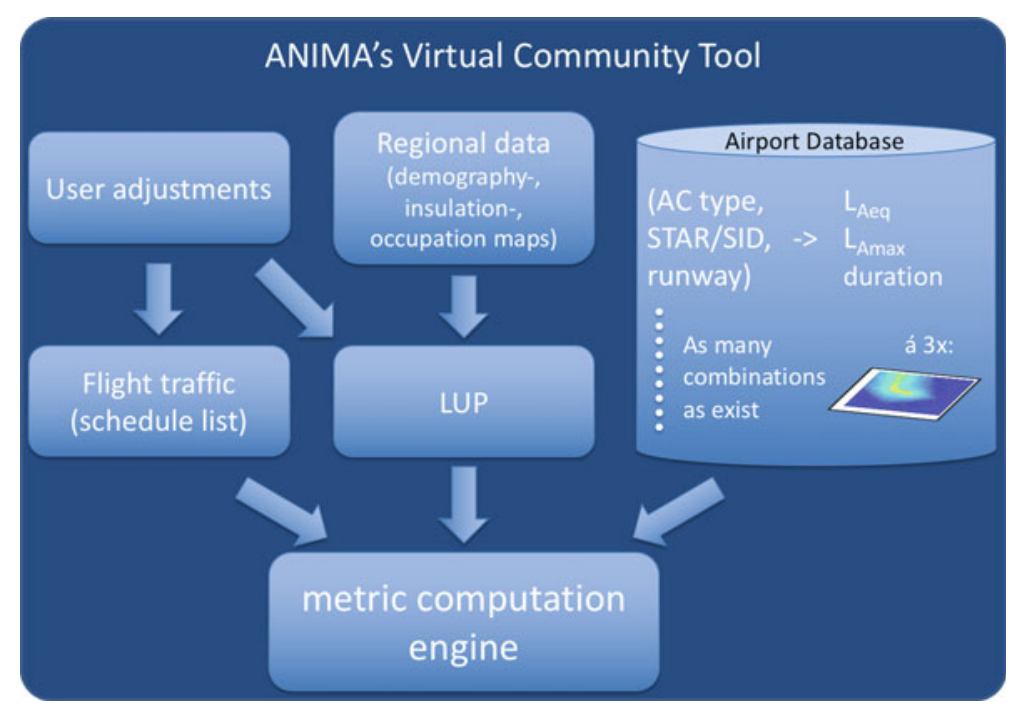

Fig. 3 Workflow of the demo VCT 
By means of the metric computation engine, the tool enables the analysis of various scenarios for one airport, i.e., a given airport database. A scenario contains the flight schedule together with the traffic modifications defined by the user as well as the land use plan (LUP) areas and the settings of the computations. The user is able to edit the traffic composition through the graphical user interface. The main idea of the tool is to allow for adjustment of the overall properties of the composition of the air traffic instead of managing single operations one-by-one. This approach allows very easy modification/redesign of the air traffic and thus facilitates the analysis of the effects of planned interventions or expected trends, such as increasing or decreasing the amount of operations on a flight path. Moreover, land use plans (e.g. financing window insulation in a certain area, or establishing a business area, etc.) can easily be defined and modified using the interactive map visualisation window.

Once a scenario is defined, the computation of the selected acoustical and nonacoustical indicators is performed. These indicators, each being defined on the geographical grid of the airport, are referred to as metrics. The computation of all metrics necessitates iterating over the flight schedule and accumulating the corresponding acoustical footprints as well as calculating non-acoustical indicators at the same time. To be able to handle long schedules containing several months of traffic in a short amount of time, the computation of each metric is specialised and the possibilities to reduce the computational burden are exploited wherever possible. The computation of acoustical indicators has been validated by comparing the results provided by our tool to reference computations carried out using commercial software packages.

The main functionality of the tool is then the analysis and comparison of the metrics computed for one or several different scenarios by means of a powerful visualisation engine. Its map visualisation window (see Fig. 4) allows for displaying the data as colormaps rendered over the satellite view of the area. The colormaps can be augmented by an arbitrary number of contours that are fully customisable by the user. At the same time, the visualised data can be exported and later imported facilitating further comparisons.

\section{Features}

The most important capability of the Virtual Community Tool is that it is able to compute various acoustical and non-acoustical indicators, including standardised quantities (e.g. $\mathrm{L}_{\mathrm{DEN}}$ or $\mathrm{L}_{\text {night }}$ ) and metrics that are customisable by the user. One example of such customisation is the ability to change the typical period of sleeping hours of people which affects the calculation of the awakening indicator.

Also a key, unique feature of the tool is that it enables the user to modify the global properties of the traffic of the airport through a clean graphical user interface that is easy to handle. In particular, the following properties of air traffic can be adjusted: 

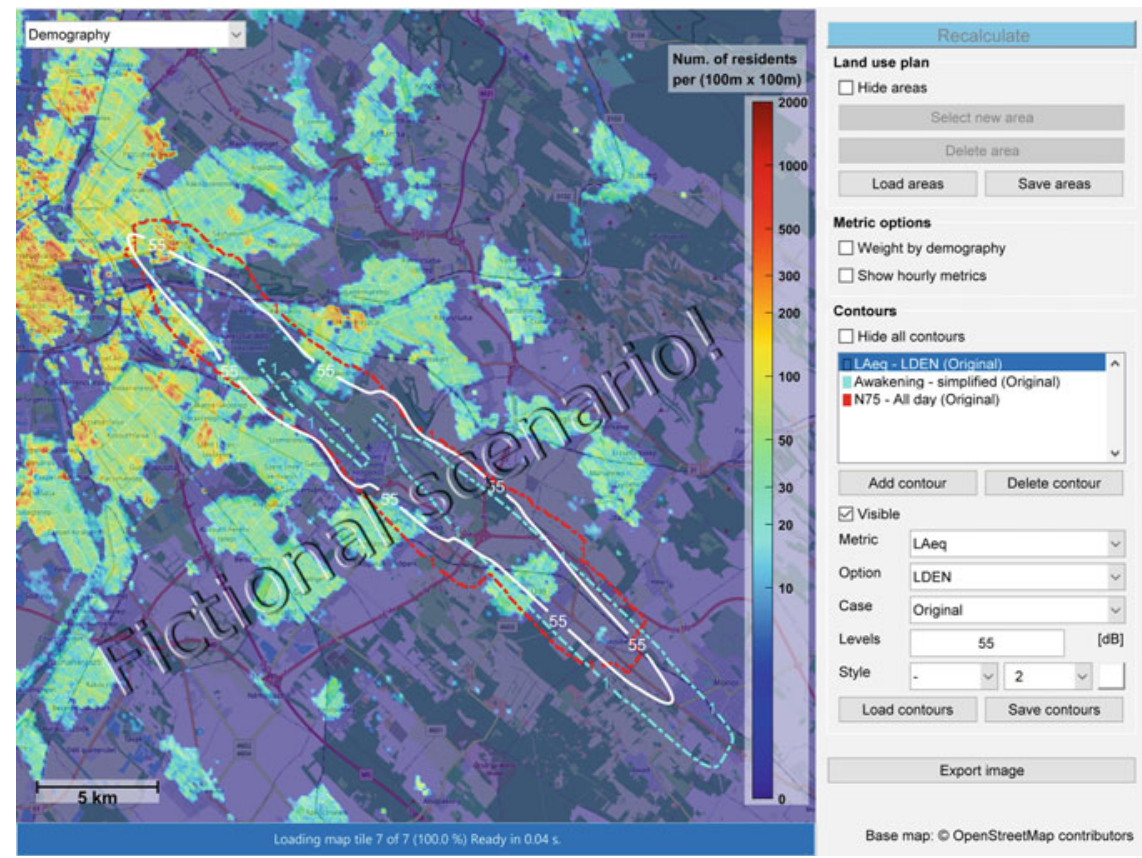

Fig. 4 Map visualization window showing various metrics for one scenario. One can observe that areas defined by crucial limits of the metrics mark out somewhat different areas

- The global and the hourly amount of operations can be modified, meaning that the traffic as a whole can be increased or decreased. Furthermore, the traffic can be reorganised among the hours of the day, for example by moving a certain percentage of the traffic from one hour to one or many others. This feature is particularly useful for examining the day-evening-night balance of the noise and annoyance caused by the current or the planned air traffic.

- The usage of runways and flight paths, allowing the adjustment of the amount of traffic on them. Besides the ability of foretelling the effects of introducing new flights, this feature can be especially useful for predicting the change of indicators by such events as a renewal of a runway.

- The composition of the fleet, i.e. the relative amount of different types of aircraft, allowing the replacement of older aircraft types by newer ones, as well as completely banning operations of given aircraft types. This functionality allows for forecasting the effect of the renewal of the fleet of airlines.

Areas for various types of land use plan actions may be defined by the user by simply marking them on the map. Area types include green parks, business areas, university campuses, or areas where window insulation for the houses is funded. The areas defined by the user affect the computation of both acoustical and non-acoustical indicators. 
If a demographic map of the examined area is available, the VCT imports it automatically. Once the demographic map is loaded, the acoustical and annoyance metrics can also be visualised taking the number of affected people into account, indicating the seriousness of possible aircraft noise related problems. Furthermore, the demographic map may be supplemented by a so-called occupancy map, which describes - on an hourly basis — what percentage of the habitants are in fact at home. This enables taking the number of affected people dynamically into account. This unique feature is also exploited in defining the land use planning for the vicinity of the airport. As an example, it can be expected that a business area or a shopping center is not inhabited during the night hours.

The user has the possibility to either perform a series of adjustments on one scenario, resulting in a modified scenario or to perform different adjustments on the same scenario and then to save them as separate scenarios.

The integrated map visualisation window allows for a straightforward comparison in both cases: the modified scenario to the original, hence enabling a quick overview of the effects of the planned changes or the comparison of various options for a given starting situation. Moreover, the capability to compare several scenarios also allows for comparing different schedules, i.e. worse day versus long-term average or preferred versus non-preferred oparation mode of the airport.

\section{Scenario Show-Cases}

Let us recall a statement from European Parliament [4]:

"Furthermore, the use of new metrics like Number of Events above a certain noise value are being pushed forward. As it is indicated in the WHO 2018 Environmental Noise Guidelines for the European Region "There is additional uncertainty when characterising exposure using the acoustical description of aircraft noise by means of Lden or Lnight. Use of these average noise indicators may limit the ability to observe associations between exposure to aircraft noise and some health outcomes (such as awakening reactions); as such, noise indicators based on the number of events (such as the frequency distribution of LA,max) may be better suited. However, such indicators are not widely used".

There is, therefore, a proposal to start giving more priority to other noise indicators (in particular event-related metrics) as well as calculating lower noise level contours to present noise exposure, which is a challenging modification considering the way the noise effects have been studied until now.

This also supports the notion that annoyance is not just a yearly value and cannot be characterised by a single metric. More and more countries are considering various metrics simultaneously. Here, a good software comes in handy especially for "starting the journey" airports, i.e. airports with less practice in aircraft noise abatement.

In the following you will see several scenarios demonstrating the capabilities of the demo Virtual Community Tool. By showing differences in contour-sizes, we definitely don't want to give a position on what is appropriate to use. We just want 

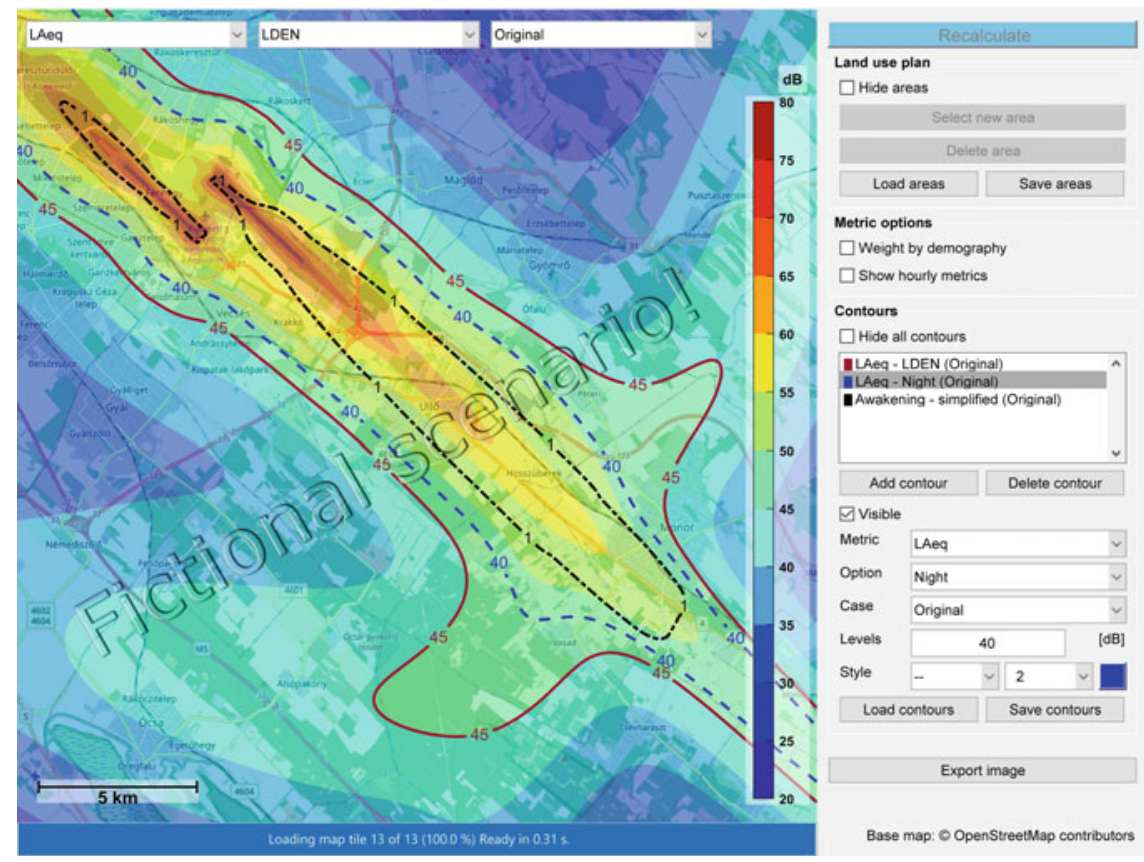

Fig. 5 Multiple metrics can be shown at the same time. Color mapping depicts $L_{d e n}$, brown continuous contour shows the limit for $\mathrm{L}_{\mathrm{den}}=45 \mathrm{~dB}$, blue dashed curve shows $\mathrm{L}_{\text {night }}=40 \mathrm{~dB}$, while the black dash-dotted line encloses the area with $>1$ additional awakening per night

decision makers to be in a better position to know what to expect. This information could be used for communication purposes or taking actions.

\section{Multi-metrics evaluations}

State-of-the art research suggests the definition of protection zones based on several properties, not just one. For aircraft noise, WHO recommends limiting the aircraft noise exposure to less than $45 \mathrm{~dB} \mathrm{~L}_{\mathrm{den}}$, and for $\mathrm{L}_{\text {night }} 40 \mathrm{~dB}$ [5]. Another recommendation is to keep the average additional awakenings induced by noise below 1 per night [6]. As our demo VCT is capable of computing and showing several metrics at the same time, one can clearly observe what areas should be protected to fulfill all three conditions (See Fig. 5.)

\section{Sleep-time preference}

People differ from each other. Some prefer to go to bed later and also get up later, others go earlier to sleep and get up also earlier. With the ability to flexibly change the sleeping hours of people, independently from the night period defined in regulations by each country, with our tool one can observe that "late sleepers" in a much larger area have their nights unprotected from being woken up more than once in average by the air traffic (see Fig. 6.) This example definitely shows 

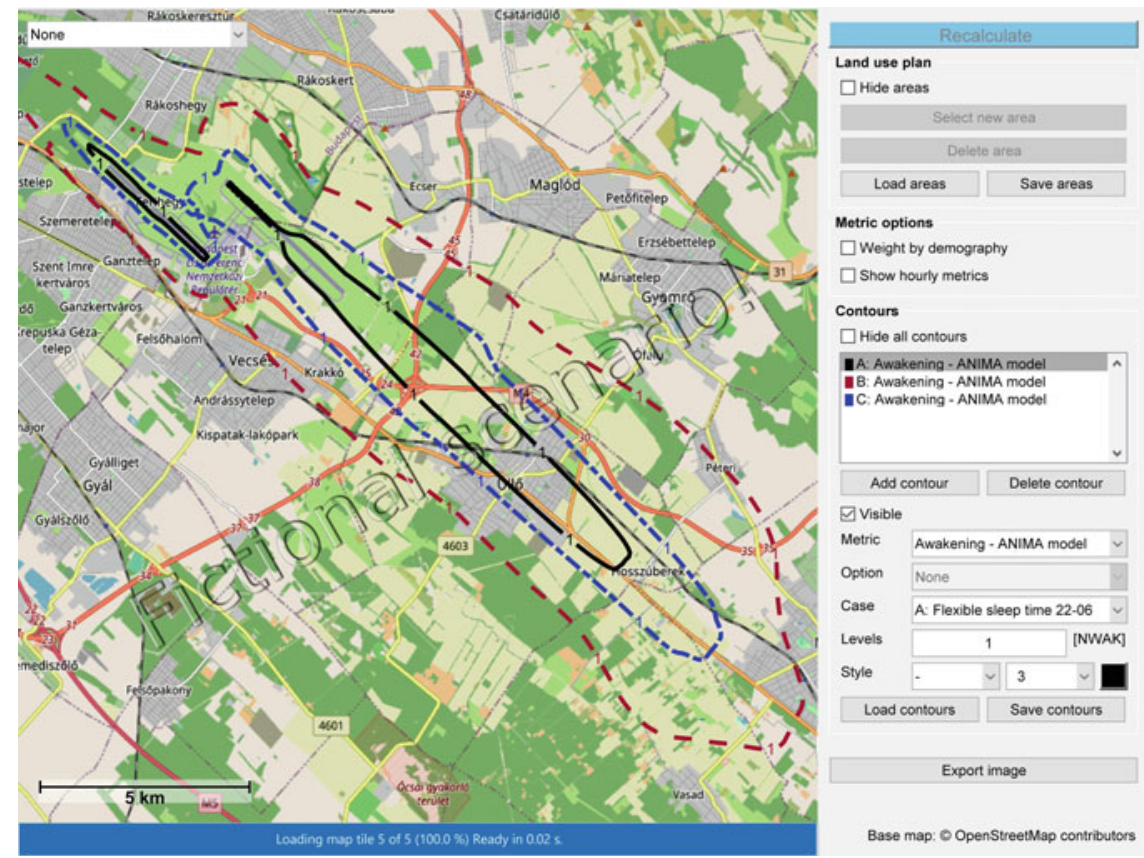

Fig. 6 More than one additional awakening per night area for people sleeping from 22-06 (black curve) and for those sleeping from 23-07 (brown dashed curve). Blue dash-dotted curve shows these latter sleep hours with the reorganisation of the morning traffic: $50 \%$ of the flights between 06-07 are moved to $07-08$

that while it is not realistic to completely shift the "airport start" to one hour later, it is an option to shift at least some flights to after 7 o'clock, or in case there is budget for it, to extend window insulation programs to a larger area, or finally at least to spread simply the knowledge that "late-sleepers should not live near airports".

\section{Scenarios to expect}

It could be preferable to consider at the same time a long-term average and some kind of maximum operation. Especially normal, but non-preferred configurations could be cause for complaints, because published maps often present only the long-term average, lowering those less-frequently happening, inconvenient levels of areas, which receive high noise load only during non-preferred times. The effect is even stronger when preferred and non-preferred configurations are considered. It is worth explaining to people by visualisation why they sometimes feel so bad about the noise: because after a period with favourable conditions the contrast to the unfavourable is much more pronounced. However such scenarios are computed from completely different flight schedule lists. This is 

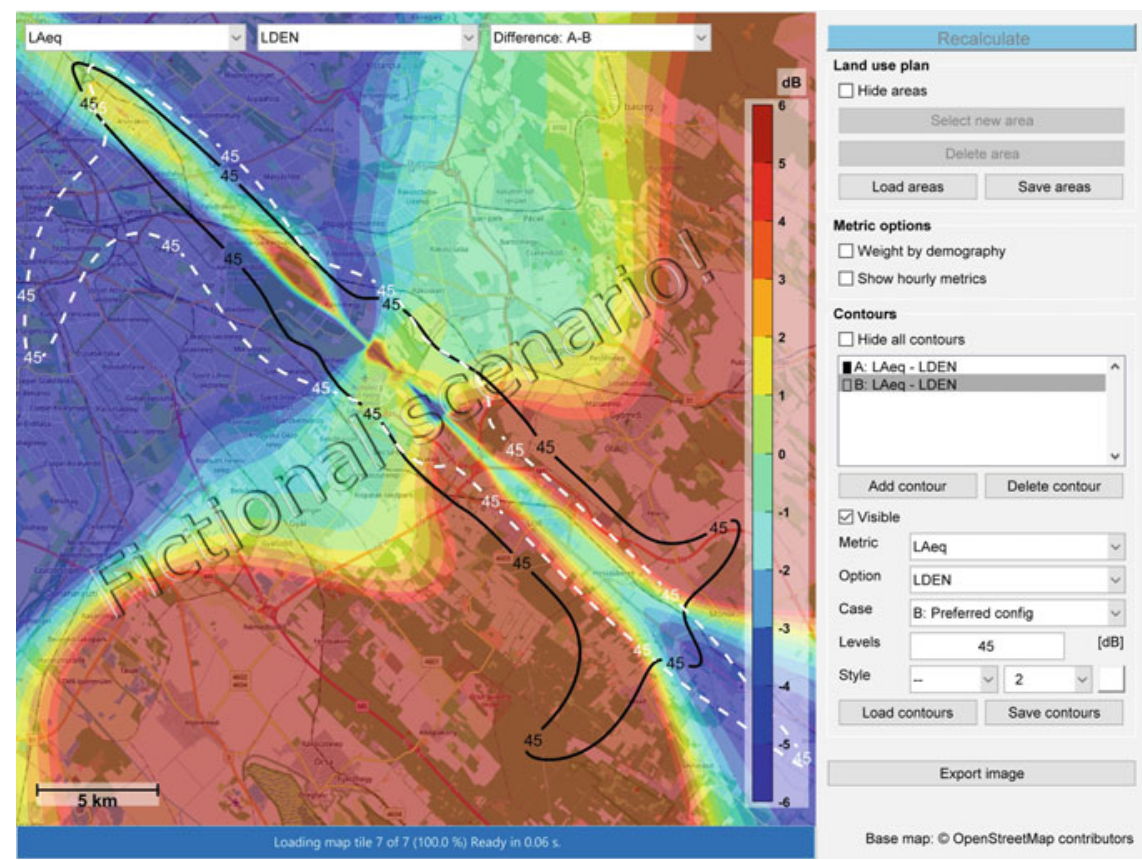

Fig. 7 The colormap shows the level difference of the non-preferred versus the preferred configuration. Although the strongly affected area (red area, $>6 \mathrm{~dB}$ ) is huge, beyond the $45 \mathrm{~dB}$ contour curves the noise is overall quite low. Still, the area between the two contour lines is remarkably large. (Black curve is the non-preferred, white dashed curve is the preferred configuration.)

not a problem for the VCT, as the intelligent visualisation engine is able to present several scenarios on the same map. (See Fig. 7).

\section{Future}

During Land Use Planning, it is wise to look a bit towards the future. While nobody can tell what will actually happen, most airports already have experience in evaluating their flight traffic over the years. Most probably the increase in flight operations and the renewal of airlines' fleets can be estimated. To compute such expectations is really easy for the user: just the increase in the total number of flights need to be changed, and a few replacements of some older, but frequently used aircraft types by some current ones and voilà, one can have an idea how the airport's footprint will perhaps evolve in the upcoming years. Figure 8. depicts such an estimation.

\section{Critical Hours}

We know from experience that some hours are more critical than others, e.g. falling asleep is more prone to disturbance by noise events then when one is already asleep. So people could be interested in traffic during these hours. As our Virtual Community Tool performs internal computations on an hourly basis, the Map Display Window 

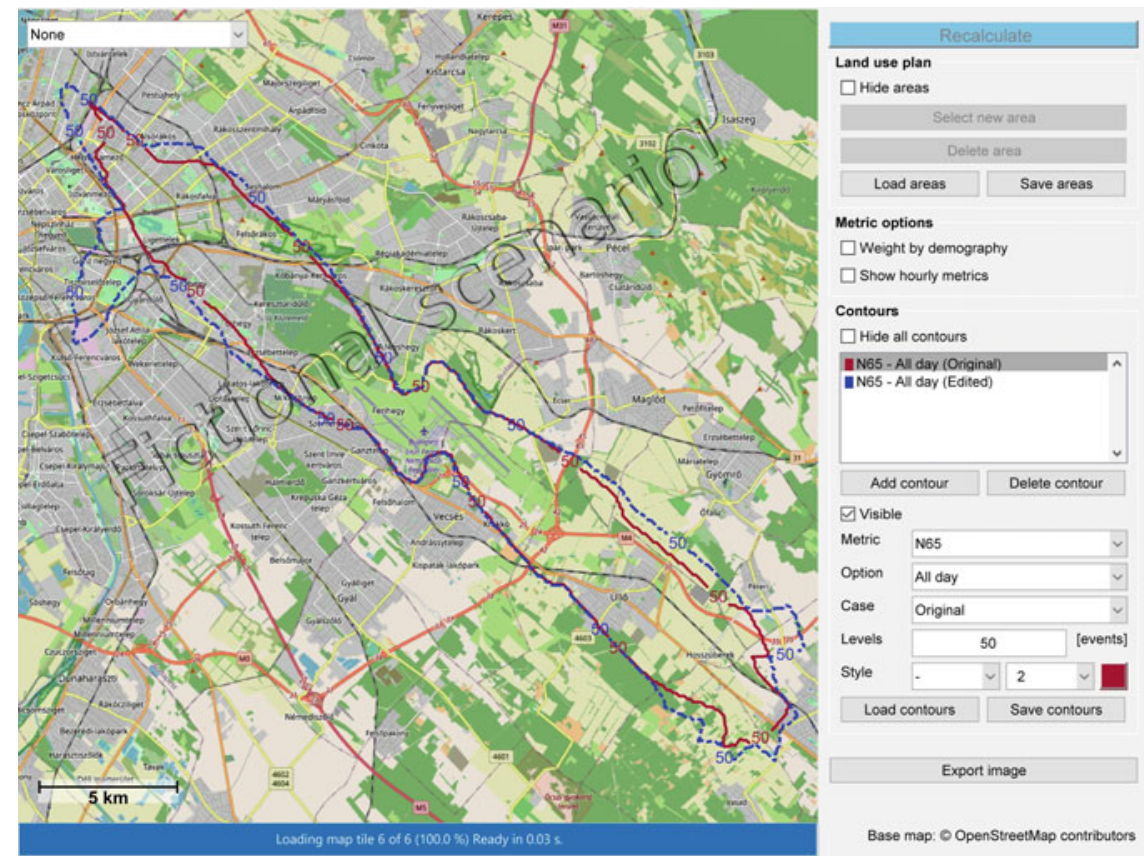

Fig. 8 Actual state (brown curve) versus 25\% increased traffic with upgrading 20\% of the older part of the fleet by newer aircraft (blue dash-dotted curve). Contour curves show 50 movements per day with a maximum noise level above $65 \mathrm{~dB}$ (N65)

allows the presentation of metrics for specific hours over the map but also the overlaying of contours for several hours. (See Fig. 9).

\section{Land Use Planning}

Land Use Planning is a powerful way to control noise annoyance. Building well soundproofed business-areas or shopping centers near airports could be examples for it. In these areas no population is there to be disturbed during the night time, and during the day, business areas can afford to pay for well soundproofed buildings, while in shopping centers the noise levels are usually already so high indoors that higher outside noise levels are not relevant. But also the effect of financing window insulation in a certain area is worth studying, especially if we know the typical original sound insulation quality of houses and the seasonal habit of people to close or leave their windows open during the nights. The VCT allows for an easy definition of land use planned areas by defining simply their functionality. Also a map containing the typical sound insulation quality of houses around the airport can be used by the tool, so the effect of soundproofing improvement can be easily studied. On Fig. 10 an example scenario is shown: some areas received window insulation and a business center has been established near the airport. 

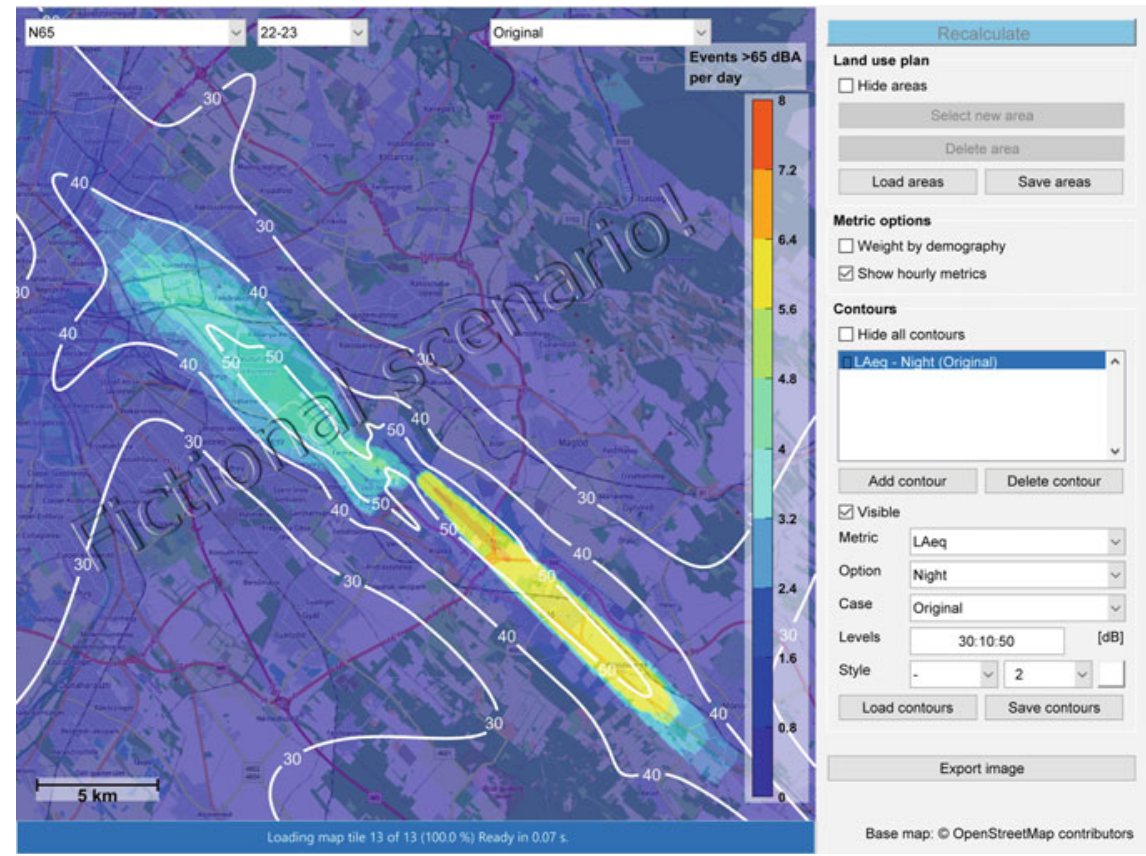

Fig. 9 This figure shows the overall night-time's noise load with white contour curves. While the average noise-load towards the city is similar to those away from it, actually the number of loud events depicted by the colormap (maximum noise level above $65 \mathrm{~dB}$ ) between $22-23$ o'clock is about half as in the opposite direction. This is a favourable effect for the densely populated city area

\section{Dynamic Noise Maps}

\section{Background and Definition}

Dynamic noise mapping represents a relatively new concept in the airport noise literature. Different authors have used this term for various purposes in the previous years. Before going into further details, it is beneficial to compare the current usage of this term and to precisely define the meaning of the "dynamic noise maps" concept in this book.

Most of the research studies have used this term to present noise in a given moment of time, i.e. to differentiate between noise maps for different period of the day (peak or off-peak hours) and for the different days of the week (weekdays and weekends) [7, 8]. Another research project [9] defines dynamic noise maps as acoustic maps that illustrate in real time the temporal change of noise levels. Such noise maps are constantly updated using algorithms and software in real time for different operating conditions (sources, traffic, and weather conditions), by detecting 

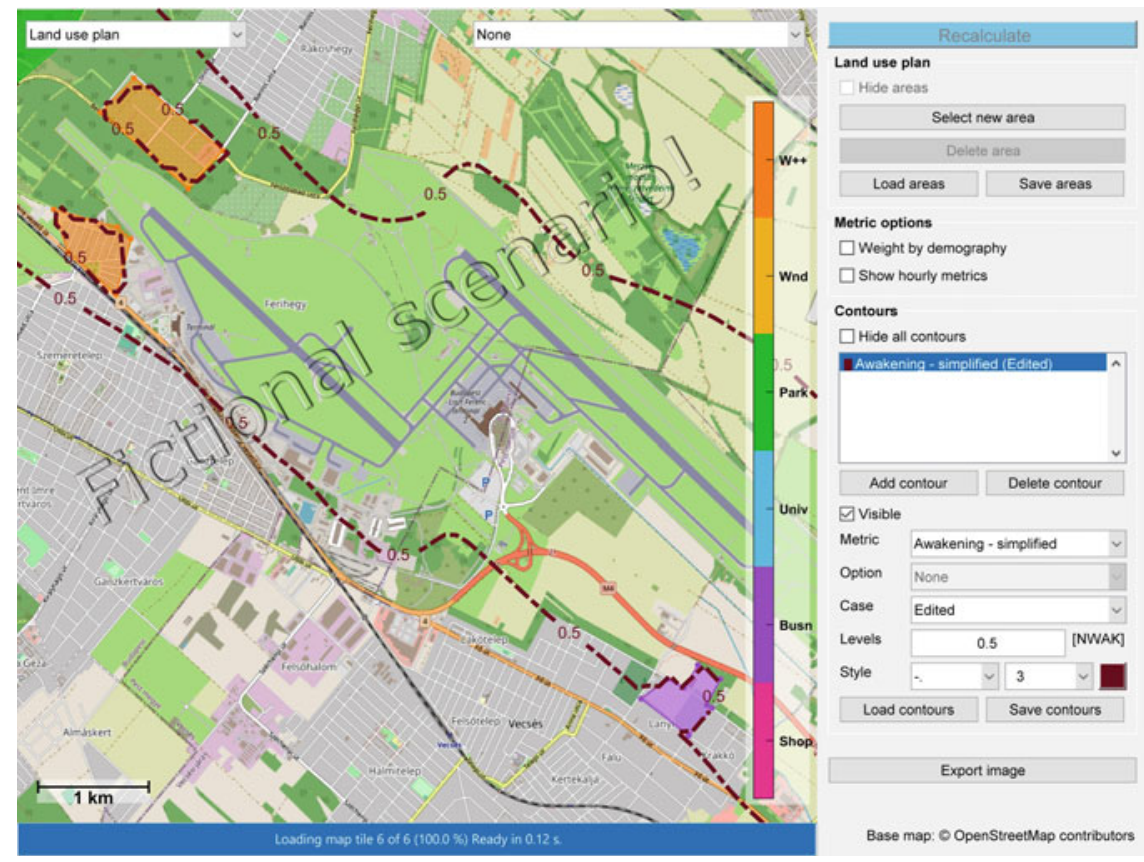

Fig. 10 Awakening is reduced in areas with LUP functionality. Purple marked area depicts a business area, while the orange ones define window insulation programs

noise and meteorological data from low-cost monitoring stations and weather sensors [10].

When real-time noise levels obtained from noise monitoring stations are used for dynamic noise mapping, measurements could be taken only at specific points due to limitations in the number of noise monitors. To obtain the existing noise levels for the rest of the area of interest some estimates are needed. This is usually done by updating the previously calculated noise levels using a reverse engineering method (based on a sound power assigned to each existing noise source and the distance to the measuring point) [11].

In several research studies, production of dynamic noise maps has been performed by including the citizens into the process of collecting the noise levels data in their surroundings instead of using noise monitoring stations. In that sense, citizens act as sensors and measure the level of noise using applications on their mobile phone or some other smart device [12-14].

Although the ultimate goal of making any noise map should be to determine the number of people exposed to noise, none of the mentioned approaches consider the dynamics of population movement. Even though such dynamic noise maps indicate different noise levels during the observed periods for which they are made, it is assumed that the population is constant in all observed locations, which is not the case in reality. 
The first attempt to include the dynamics of a population's movements into the assessment of aircraft noise exposure was carried out by Ganić and Babić [15], followed by a series of papers by Ganić et al. [16-18] and Ho-Huu et al. [19]. In all these research efforts, the emphasis was on optimising the aircraft assignment to departure and arrival routes, while dynamic noise maps were created only for one-day scenarios to demonstrate the possibilities of the developed algorithm to reduce noise annoyance and fuel consumption. Furthermore, the calculations of daily population mobility included many assumptions and were based only on data from the census.

In this book, different ways of collecting human mobility patterns will be explained in more detail along with the methodology used to incorporate it into dynamic noise maps. Furthermore, a real case study conducted within the ANIMA project, based on the one-year air traffic data, will shed light on the benefits of using this new approach and demonstrate how daily movements influence the estimated population noise annoyance around an airport.

\section{Human Mobility Patterns}

In the sense of dynamic noise mapping, human mobility patterns (sometimes also referred to as population daily mobility or movement patterns) are defined as the movements of human beings (individuals as well as groups) in space and time. Motivation behind people's movements on a daily basis is manifold. While most common daily trips include commuting to and from work or school, they are also connected with the social, leisure and other activities.

During the last decade, substantial progress has been made in the study of human mobility. Not only by the significant advancement in the field of information and communication technologies enabling more accurate tracking of people's movements, but also in that the collection and processing of such data is more accessible to the general public.

While geography might be the first discipline to analyse mobility data and put forward corresponding theories to describe travel patterns [20], the study of human mobility currently spans several disciplines. It is widely used in transportation studies to describe how people plan and schedule their daily travel, as well as to provide better forecasts of future travel patterns.

A better understanding of human mobility patterns leads to more appropriate urban planning and infrastructure design, new tools to monitor health and well-being in cities, reduction of pollution, internal security and epidemic modelling, to name but a few. In this book, the special emphasis will be given on the use of human mobility patterns to estimate more accurately the number of people annoyed by aircraft noise. 


\section{Different Ways of Collecting Human Mobility Patterns}

Collection of information on human mobility has a long tradition. Some of the wellknown and widely used techniques to collect the data include surveys and questionnaires. In particular, census data, collected periodically through national surveys, contain the questions related to the location of the workplace and school/faculty as well as the place of residence. By creating an origin-destination matrix, these data can be used to estimate daily/weekly commuting flows within a city or on a country level.

Another way of using questionnaires for collecting the mobility data is by conducting National travel surveys which have proved to be valuable for modelling and planning of transport systems. Compared to the census data, travel surveys contain more detailed information about daily activities of persons participating in the survey, including number of trips per day, origin and destination of each trip, time of the beginning of each trip, travel time and distance of each trip, purpose of a trip, mode of transport, etc. By using proven statistical methods, data collected through travel surveys allow us to simulate the movements of the whole population with great detail.

Due to technological development and the increase in usage of the internet, the methods to obtain the data have changed through the years, though the purpose mainly remained the same. Digital footprints produced by people using various digital services such as mobile phones, smartphone applications, or social networks, could provide valuable insights into their daily movement patterns.

These digital footprints can be classified as passive and active [21]. The main difference between them is whether they are left voluntary or involuntary. Many online activities such as tweeting or tagging a photo carry an information (electronic trail) about the location and timestamp that could be used to track the movement of the users when the frequency of such activities is satisfactory. Passive footprints are collected involuntary by using smart-card data, mobile phone records, GPS data, while active footprints come from the users themselves when they expose locational data in photos or messages while using social networks such as Twitter, Facebook of Foursquare and photo-sharing web sites, like Flickr or Instagram.

All these methods can be used to collect population mobility data necessary to develop dynamic noise maps. Nevertheless, numerous challenges can be encountered due to privacy issues. To protect the anonymity of individual persons or businesses, any data must be collected in accordance with the legislative framework, such as the General Data Protection Regulation (GDPR) that harmonises data privacy laws across Europe. Some of the measures can include signing a Confidentiality agreement and Declaration on Data Protection by the person who will use such sensitive data. 


\section{Methodology}

To be able to assess the daily mobility patterns of the population, as a first step suggested here is development of an adequate travel model. Some broad types of models used in transportation planning include the following [22]: sketch-planning models, strategic-planning models, trip-based models, and activity-based models.

The choice of model depends on the purpose for which it will be used. Because activity-based models typically function at the level of individual persons and represent how these persons travel across the entire day, they are most suitable for dynamic noise maps. Since detailed explanation of the activity-based travel models goes beyond the scope of this book, only some brief description of the main concept will be given herein. For more details, interested readers may refer to [22].

\section{Activity-Based Travel Models}

Activity-based models consider activity and travel choices for each person throughout the entire day, taking into account different types and priorities of the activities that individuals are participating in [22]. The structure of activity-based models varies in the literature. Nevertheless, as shown in Fig. 11, between the model inputs and outputs, most activity-based models include the following major types of components [22]: synthetic population, longer-term and mobility choices, daily activity patterns, tour and trip details, and trip assignment.

The first component, synthetic population, represents a basis for predicting the behaviour of the households and persons in the modelled area. It contains anonymous microdata with the appropriate variables and granularity, statistically equivalent to the actual population of interest, to serve as input for micro-simulation agent-based models.

Next component involves modelling of choices that are made on a less frequent, longer-term basis, such as where to live, work or study. In addition, decisions whether to own a car, driving license, bicycles or transit passes also belong to longer-term and mobility choices that can significantly influence the availability and attractiveness of different location, mode, and scheduling choices that create daily activity and travel patterns. These choices are simulated for each agent in the synthetic population.

Conditional upon predicted longer-term and mobility choices, all travels during the day are simulated using day-pattern, tour-level, and trip-level models. The main

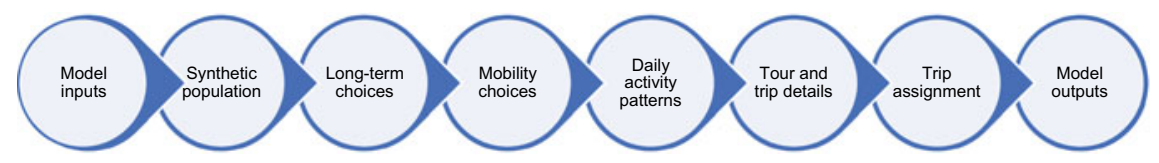

Fig. 11 Activity-based travel model components [22] 
output of the daily activity pattern model is the exact number of tours that each individual makes for each of a number of different activity and tour purposes. Scheduling, location choice, and mode choice are all relevant at both the tour level and the trip level.

Scheduling model within the tour predicts the departure and arrival times for mandatory purposes (such as work or school), while the trip-level models simulate additional stops within the tour, for example on the way home, for other non-mandatory purposes (such as shopping, social visits, recreation, etc.).

Apart from the usual home and work/school locations that are predicted within the longer-term and mobility models, location model is used to predict the location of any intermediate stops along the tour, as well as tour origin and the tour destination.

Mode choice model predicts the use of mode at both the tour level and the trip level. In most cases, people are inclined to use the same mode for an entire tour, where the selected mode is the same for each trip within the tour. Nevertheless, there are also infrequent cases of multimodal tours which could include carpooling or park-and-ride concepts.

The final step in the activity-based travel modelling is to assign simulated trips to the networks. The whole process could be iterated to recalculate the travel times or some other parameters, if needed.

\section{Calculation of Dynamic Noise Maps}

After obtaining daily mobility patterns of population, the next step is to extract the distribution of people at desired spatial and temporal resolution. The most detailed spatial resolution would include every single location where people spend time. Nevertheless, such a detailed approach is not practical nor needed for airport noise impact studies since the aircraft noise levels do not differ significantly among closely located points. Another approach is to aggregate points into grid cells (e.g. $500 \times$ $500 \mathrm{~m}$ ) and to calculate noise levels only for grid cell centroid which will then represent all the points within that cell.

On the other hand, temporal resolution will depend on the change in number of people at different locations and frequency of activities in the observed model. The minimum temporal detail should include at least four or five time periods in the day, as opposed to some models that use continuous time (e.g., 1,440 one-minute periods in the day). Furthermore, temporal resolution could be observed separately for working and nonworking days since population daily mobility patterns can be completely different from each other.

The noise metric that needs to be calculated for each location is LAeq,T or the A-weighted, equivalent continuous sound level determined over time period T. After calculating LAeq noise levels, the next step is to match the number of people exposed to those noise levels at each location (spatial resolution) during each time period (temporal resolution). As a result, cumulative noise impact for each person for the whole day could be calculated. 
For example, if the model uses 24 time periods in the study, LAeq, $1 \mathrm{~h}$ will be used, where $1 \mathrm{~h}$ denotes the one-hour time period over which the fluctuating sound levels need to be averaged based on yearly average traffic for each hour. The overall traffic for the whole year should be divided into hourly periods, separately for working days (Monday to Friday) and non-working days (Saturday and Sunday) and then averaged (divided by number of days which will be different for working and non-working days). As a result, 48 different scenarios will need to be calculated.

\section{How Do Human Mobility Patterns Influence the Population Noise Exposure Around an Airport?}

To demonstrate to what extent human mobility patterns could influence population noise exposure, let us observe an example of Heathrow airport and work-related commuting patterns to and from one local authority. London Borough of Hounslow, located east of the airport, is used here as an example since the largest part of its territory is situated within the Heathrow airport $\mathrm{L}_{\mathrm{den}} 55 \mathrm{~dB}$ noise contours.

Figure 12 shows work-related commuting from and to (number shown in brackets) Hounslow for each local authority around the airport. According to the 2011 UK Census, there are 102,654 residents aged 16 and over in employment living in Hounslow. By analysing the location of usual residence and place of work, it is detected

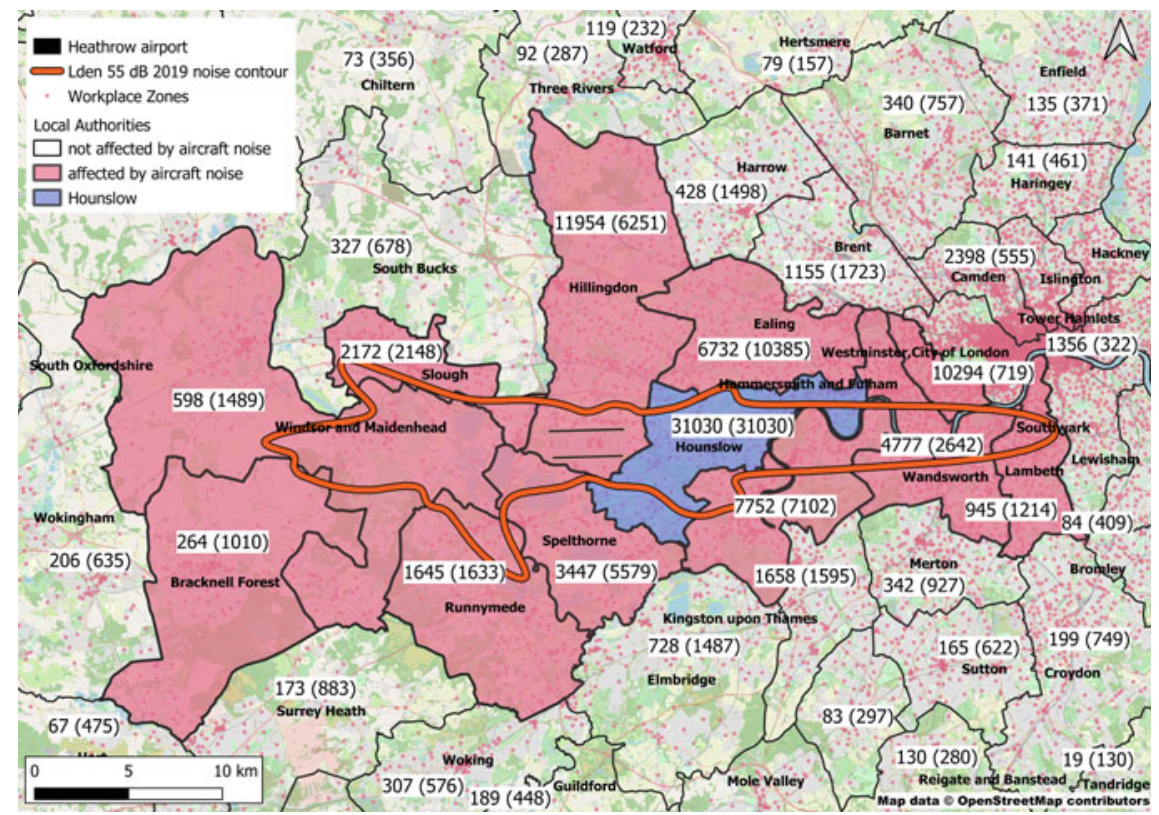

Fig. 12 Work-related commuting from (to) Hounslow 
that only $30 \%$ of them $(31,030)$ work in Hounslow, while the rest of the residents work in other local authorities. This implies that for $70 \%$ of the working population within this area (71,624 residents), the noise exposure could be incorrectly estimated (probably overestimated) since they will be spending a large portion of the day (at least while during their working hours) outside of their usual residence. For example, 11,954 residents of Hounslow (11.64\%) work in London Borough of Hillingdon, while 10,294 (10.03\%) of them work in City of Westminster and City of London which are outside of the $\mathrm{L}_{\mathrm{den}} 55 \mathrm{~dB}$ noise contours. There are 43,730 residents who commute from Hounslow to 12 local authorities that are also, at least partially, affected by aircraft noise. This indicates that more than half of Hounslow employees work further away from the airport, in the areas not affected by aircraft noise, thus being exposed to less noise than anticipated based only on the residential location.

On the other hand, Fig. 12 also shows that, out of 105,007 employees working in Hounslow, $70 \%$ of them $(73,977)$ live outside this local authority. Most of these employees $(59.2 \%)$ travel from local authorities where the aircraft noise levels are considered insignificant, since only 42,831 of them (40.2\%) are residents of 12 local authorities affected by aircraft noise, such as nearby Ealing $(10,385)$ and Richmond upon Thames (7102).

When combining the employees traveling to and from Hounslow, this work-related commuting results in the presence of additional 2353 people within this area during the working hours compared to the number of residents. Nevertheless, the main change in individual noise exposure comes from the fact that $70 \%$ of the residents will experience noise levels different from the expected one at their residential locations. The biggest difference between inflow and outflow of commuters is observed for the City of Westminster and City of London where 10,294 of residents commute from Hounslow to work there, while only 719 of their employees work in Hounslow. This is easily explained having in mind that the highest number and concentration of workplace zones is in this part of London.

The similar conclusion can be drawn from the commuting patterns of high school pupils and students, while the proximity of elementary schools and kindergartens to residential locations makes the commuting patterns of the youngest members of the society irrelevant for this kind of analysis. Apart from the trips from and to work or school, which are regarded as main or mandatory activities, there are many additional non-mandatory activities with various trip purposes that are also relevant for dynamic noise mapping. Timing when these activities occur as well as their duration also affect the noise exposure of individuals performing these activities. Results from London Travel Demand Survey, conducted by Transport for London, shown in Fig. 13, indicate that travel patterns are steady throughout the years in terms of the time of the beginning of the trip. The highest peaks, when most of the trips start, are observed from 7 to 9 AM and again from 3 to 5 PM. This is usually in correlation with the time when people leave for, and return from, work or school.

Usual time of the beginning of the trip differs significantly for different travel purposes. Figure 14 shows the results from the National Travel Survey conducted in the UK, where all the activities are combined and presented as eight different 


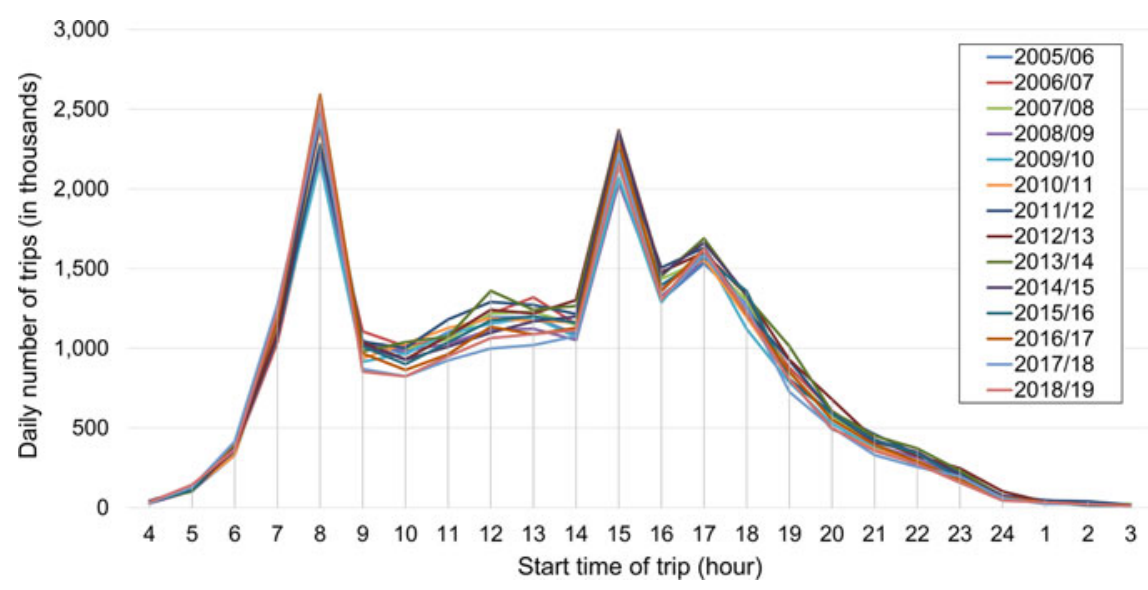

Fig. 13 Trips by start time in London (Source: London Travel Demand Survey)

purposes of trips. At a first glance, obvious differences in trip start times for different trip purposes can be observed.

Although for some trip purposes, such as business (Fig. 14b) or holiday (Fig. 14h), there is a fairly uniform distribution of trips during each hour of the day, for most of the purposes a pronounced peak can be clearly seen. For example, the largest number of employees start their journey around $6 \mathrm{AM}$, while the second peak with a much smaller number of trips occurs around 6 PM (Fig. 14a). For trips with the educational purpose (Fig. 14c) including escorting trips (Fig. 14d), there are two approximately equal peaks, around 9 AM and 4 PM. Most of the shopping activities start within the period from 11 AM to 3 PM with the moderate intensity also shown during evening hours until the midnight (Fig. 14e). As expected, only activities that include entertainment and visiting friends are dominant during late evening and early morning hours (Fig. 14g).

All these differences are considered when developing human mobility patterns for dynamic noise mapping. They influence the temporal and spatial distribution of people, thus leading to population noise exposure other than expected when the movements of residents are disregarded in the noise mapping process.

\section{ANIMA Case Study: Dynamic Noise Maps for Ljubljana Airport}

The case study that will be presented here aims to show how population movements affect the estimated number of people exposed to aircraft noise. The research was done within the ANIMA project, to demonstrate the capabilities of dynamic noise mapping compared to the traditional way of developing noise maps. More detailed 

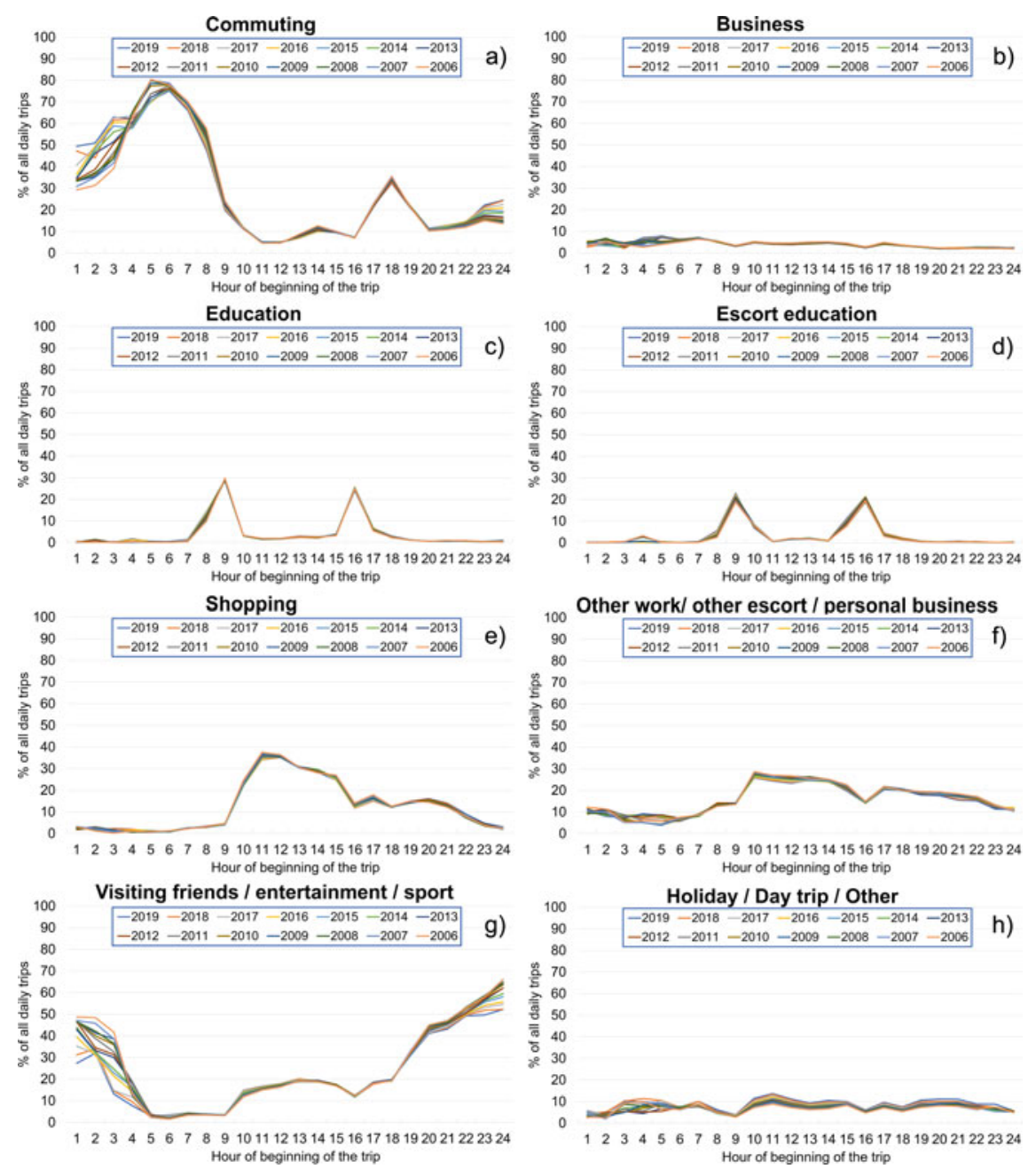

Fig. 14 Trip start time by trip purpose (source: UK National Travel Survey)

information about the methodology and input data for this study can be found in [23].

Ljubljana Jože Pučnik Airport was chosen for the case study. It is the busiest airport in Slovenia, handling more than 1.7 million passengers and approximately 31 thousand aircraft operations in 2019. The airport has a single runway $3300 \mathrm{~m}$ long and is located $20 \mathrm{~km}$ northwest of the Ljubljana capital. Even though the Ljubljana airport is not recognised as a "major airport " as defined in the Directive 2002/49/EC, proactive noise assessment actions have been undertaken by the airport authority including the development of noise contour maps and regular continuous noise monitoring in the most noise exposed areas for several years. 
The year 2018 has been selected for this case study based on the availability of data regarding population mobility patterns and air traffic. The yearly traffic at Ljubljana airport for 2018 consisted of 35,512 aircraft operations, performed by 213 different aircraft types, indicating that on average, there were approximately 97 operations per day, or 4 movements per hour. There were several peaks during the day, with the most flights occurring between 5 and 6 PM (nine flights in average), while only $6 \%$ of operations were performed during the night hours.

The population mobility patterns are assessed using a dedicated national travel survey. The most recent one conducted in Slovenia was the Daily Passenger Mobility Survey (TR-MOB 2017). The data were obtained from the Statistical Office of the Republic of Slovenia through the special request for protected microdata containing detailed information about each trip on an individual level. The number of respondents in this survey was 8,842 , while the number of trips conducted was 24,195 . Since $1355(15.3 \%)$ survey participants reported that they stayed at home, the average number of trips per day can be calculated as 2.7 or 3.2, depending on whether all the respondents are considered or only the ones conducting the trips. In terms of different trip purposes, leisure activities were the reasons behind most of the recorded trips (35.6\%), followed by commuting trips to and from work $(24.3 \%)$. Other trip purposes included education, professional and personal business, shopping, and escorting (driving/picking up/accompanying a child or other person). More information about this survey, including explanations about methodology, is contained in [24].

Once all the data had been collected, the A-weighted equivalent continuous sound level was calculated to match the number of people exposed to those noise levels at each location $(500 \mathrm{~m} \times 500 \mathrm{~m}$ grid) during each one-hour period. As a result, the cumulative noise impact for each person has been assessed and the results are presented herein.

The results lead to the conclusion that even though people live at locations enclosed in the $37 \mathrm{~dB} \mathrm{~L}_{\mathrm{den}}$ noise contour (the threshold for becoming annoyed according to [25]), $10.1 \%$ of them (marked with white square symbol on Fig. 15) are not exposed to aircraft noise levels $\left(\mathrm{L}_{\mathrm{den}}\right)$ above $37 \mathrm{~dB}$ due to daily mobility to locations away from the airport. Furthermore, apart from the 4884 people that are living within the presented noise contour (marked with red star symbol), there are additional 704 persons (14.4\%) also experiencing aircraft noise exposure (marked with yellow triangles) even though they are located outside the $37 \mathrm{~dB}$ noise contour. This can be explained by considering that people who live outside the area affected by aircraft noise may work or study within these areas at some time during the day and are, therefore, affected by aircraft noise. The fourth group of people (marked with grey circles) resides outside this noise contour and is not affected by aircraft noise, even when the daily mobility patterns are considered.

In order to better demonstrate the dynamics behind this novel approach, four different noise maps with estimated number of people at each location are presented in Fig. 16 describing: (a) night (02-03 h), (b) morning (08-09h), (c) afternoon (14-15h) and (d) evening (20-21h) periods. This figure illustrates the temporal and spatial variation in population around Ljubljana airport. In addition, the change in the number of operations between each hour is clearly visible through the different shapes and areas 


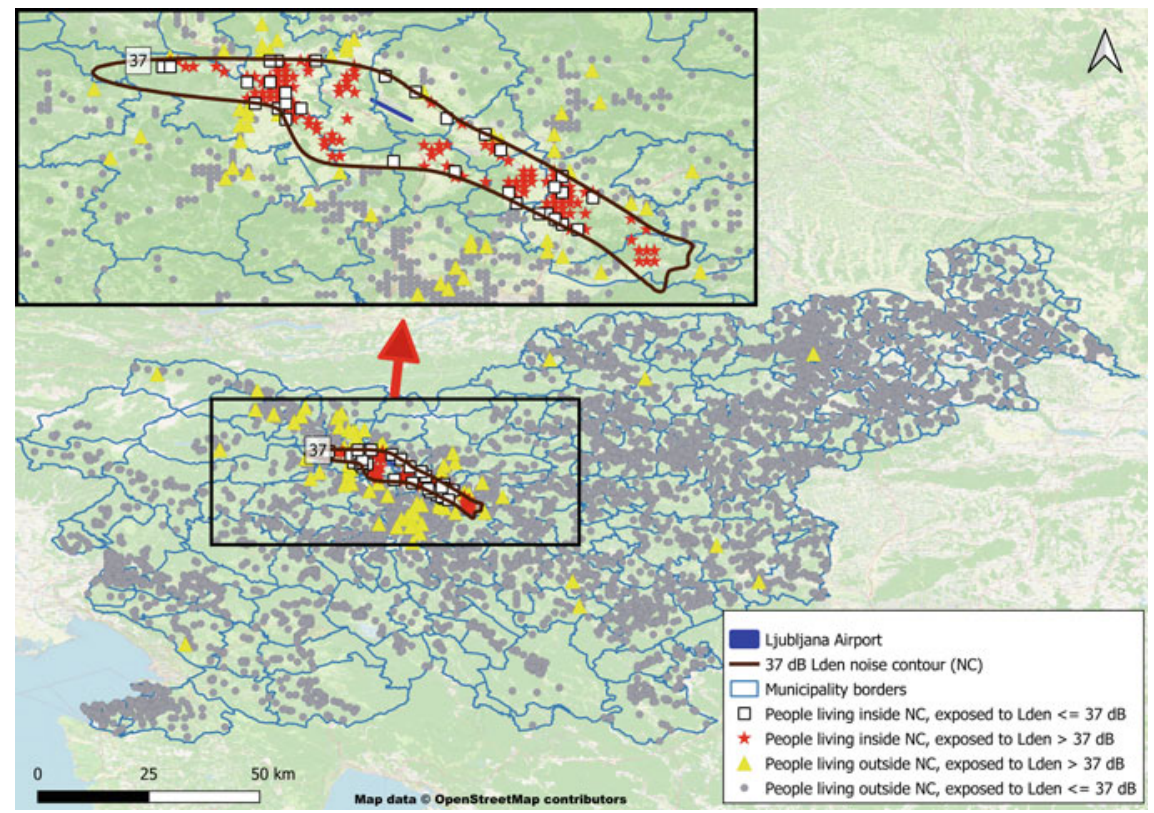

Fig. 15 Dynamic noise map for Ljubljana airport

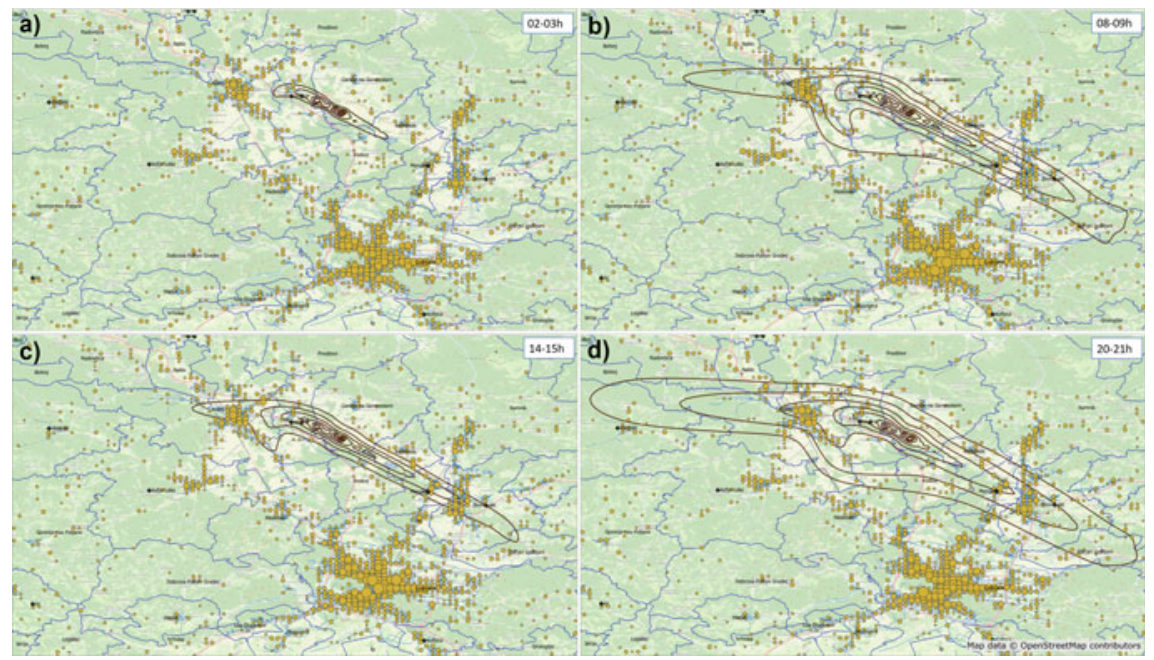

Fig. 16 Simulation of dynamic noise maps 
that calculated noise contours take. As expected, the smallest changes are observed during night hours, when the traffic frequency is low as well as the movements of the people.

It should be borne in mind that this case study only considers $\mathrm{L}_{\mathrm{den}}$ as a factor for estimating annoyance. As shown within the ANIMA project, aside from noise exposure, non-acoustic factors have a considerable influence on perceived annoyance, and they should also be taken into account in future research on dynamic noise mapping.

\section{Further Developments}

There are several directions for the future development of dynamic noise maps. This includes the involvement and contribution of several stakeholders and interested parties who can influence the collection and quality of necessary data, the adoption of legal frameworks, as well as the management of airport operations, all based on the new knowledge regarding the population noise exposure due to daily migration.

First of all, collecting more detailed data on population daily movements is the basis for a more accurate calculation of the actual exposure of the population to noise. Therefore, encouraging active public involvement, especially of the residents living in the vicinity of the airport, would significantly improve the quality of dynamic noise maps. One of the steps towards achieving this goal is to motivate citizens to contribute through participation in daily mobility surveys or by using dedicated applications that can collect data on people's movements. In that sense, it is pivotal to educate the citizens such that they could understand more clearly the needs of the airport and be more willing to participate in such endeavours. It goes without saying that by giving the necessary and truthful information about their movement patterns, residents are helping the airport to better solve the noise problem they could be faced with. On the other hand, it is also advisable to educate the airport authorities to adopt and take advantage of the new technologies and approaches through which dynamic noise maps can be implemented in practice more easily.

Although the above-mentioned surveys are mainly organised and conducted by the National Statistical Institutes, usually on a country level, one of the future directions may stimulate airports to also embrace such activities. In that regard, the airport authorities could conduct more detailed and more frequent surveys on a smaller sample, that could primarily include settlements around the airport that are most affected by aircraft noise. In that way, the movement habits of the local residents could be determined more accurately. Estimation of the number of people highly annoyed by aircraft noise, with such new data, could give the airport authorities a new perspective on the noise issue around the airport. Through such dedicated questionnaires, residents could also express their preferences about the periods of the day when the aircraft noise bothers them the most, or vice versa to indicate to the airport when, due to the nature of their activities, noise is not an issue since they could be far away from their residences. If applicable, airports could use such 
information when negotiating with the airlines about the seasonal schedule in order to reduce annoyance. Certainly, this approach is in line with the modern aspirations of every airport that has a noise issue since the current focus is primarily on improving communication with the residents, sharing information, and providing transparent reporting about the airport noise to the general public.

Air navigation service providers (ANSPs) could also benefit from dynamic noise maps and use them to reduce the adverse impact of noise on the population. The number of people in an area is a vital indicator for the noise impact analysis and should therefore be considered when making decisions regarding air traffic assignment that influence the noise allocation. Population noise exposure reduction can be achieved by optimising the distribution of aircraft on arrival and departure routes, by considering spatial and temporal variations in the number of inhabitants in the settlements around the airport, since these data are available in dynamic noise maps. One of the future developments of this approach could lead to the inclusion of dynamic noise maps into a decision support tool that could help air traffic controllers in their activities either on tactical, pre-tactical or strategic level. There are several ongoing research efforts in this direction which will allow the ANSPs to minimise the number of people exposed to noise while using the benefits of dynamic noise maps.

Finally, to apply the presented dynamic noise maps approach globally, it is pivotal to involve the policymakers who have the power of setting and directing regulatory frameworks that should follow the developments in this area. All current studies conducted on the basis of a legally imputed obligation, as is the case of strategic noise maps, consider the noise level on the most exposed façade of the building. All reported numbers of people exposed to noise are attributed to all persons living in the buildings according to the census data, regardless of their actual location. Future regulatory developments should consider the inclusion of population daily mobility patterns in the noise mapping process in order to assess the impact of noise on the population more realistically. Therefore, it is key to inform the policymakers about the possibilities of dynamic noise maps and their advantages compared to traditional noise maps currently in use. More detailed research on this topic should provide guidelines to the policymakers on how to incorporate this approach into the legislation so that any airport that has a noise problem could benefit from a dynamic noise mapping approach.

Acknowledgements Figures 4, 5, 6, 7, 8, 9, 10, 12, 15 and 16 are using OpenStreetMap data. OpenStreetMap ${ }^{\circledR}$ is open data, licensed under the Open Data Commons Open Database License (ODbL) by the OpenStreetMap Foundation (OSMF). https://www.openstreetmap.org https://ope ndatacommons.org

\section{References}

1. Report on Standard Method of Computing Noise Contours around Civil Airports (2019) ECAC Doc 29-4th Edition (7 December 2016). Downloadable from www.ecac-ceac.org 
2. van Oosten N (2004) SONDEO: a new tool for airport noise assessment. InterNoise 2004, Prague, Czech Republic, August 22-25

3. ICCAN (Independent Commission on Civil Aviation Noise), July 2020: A review of aviation noise metrics and measurement. https://iccan.gov.uk/iccan-review-aviation-noise-metrics-mea surements/

4. EU parliament (2020) Impact of aircraft noise pollution on residents of large cities. Policy Department for Citizens' Rights and Constitutional Affairs, https://www.europarl.europa.eu/ RegData/etudes/STUD/2020/650787/IPOL_STU(2020)650787_EN.pdf

5. WHO (2018) Environmental Noise Guidelines for the European Region. World Health Organisation, ISBN 978928905356 3. https://www.euro.who.int/_data/assets/pdf_file/0008/383 921/noise-guidelines-eng.pdf

6. Basner M (2008) Aircraft noise effects on sleep: substantiation of the DLR protectionconcept for airport Leipzig/Halle. In: Proceedings of 9th international congress on noise as a public health problem (ICBEN) 2008, Foxwoods, CT, pp 772-779

7. Mishra RK, Nair K, Kumar K, Shukla A (2021) Dynamic noise mapping of road traffic in an urban city. Arabian J Geosci 14(2). https://doi.org/10.1007/s12517-020-06373-9

8. Kozielecki P, Czyzewski A (2008) An application for vector-based dynamic noise maps generation. In: Joint Baltic-Nordic acoustics meeting

9. DYNAMAP (2014) [Online] Available http://www.life-dynamap.eu/project/

10. Benocci R et al. (2019) Dynamic noise mapping in the suburban area of Rome (Italy). Environments 6(7). https://doi.org/10.3390/environments6070079

11. Simón Otegui L et al. (2019) Dynamic Noise Map based on permanent monitoring network and street categorisation. In: INTER-NOISE and NOISE-CON congress and conference proceedings, vol 259(2), pp 7270-7281

12. Poslončec-Petrić V, Šlabek L, Frangeš S (2016) With the crowdsourced spatial data collection to dynamic noise map of the city of Zagreb. In: International symposium on engineering geodesy SIG 2016, pp 411-423

13. D'Hondt E, Stevens M, Jacobs A (2013) Participatory noise mapping works! An evaluation of participatory sensing as an alternative to standard techniques for environmental monitoring. Pervasive Mob Comput 9(5):681-694. https://doi.org/10.1016/j.pmcj.2012.09.002

14. Drosatos G, Efraimidis PS, Athanasiadis IN, Stevens M, D’Hondt E (2014) Privacy-preserving computation of participatory noise maps in the cloud. J Syst Softw 92(1):170-183. https://doi. org/10.1016/j.jss.2014.01.035

15. Ganić E, Babić O (2017) Air traffic assignment to reduce population noise exposure: an approach incorporating human mobility patterns. In: 21st Air transport research society world conference

16. Ganić E, Babić O, Čangalović M, Stanojević M (2018) Air traffic assignment to reduce population noise exposure using activity-based approach. Transp Res Part D: Transp Environ 63:58-71. https://doi.org/10.1016/j.trd.2018.04.012

17. Ganić E, Ho-Huu V, Babić O, Hartjes S (2018) Air traffic assignment to reduce population noise exposure and fuel consumption using multi-criteria optimisation. In: Proceedings of the 26th international conference noise and vibration, pp 69-76

18. Ganić E, Babić O, Čangalović M, Stanojević M (2017) Air traffic assignment to reduce population noise exposure: an approach incorporating human mobility patterns. In: XLIV International symposium on operational research, pp 746-751

19. Ho-Huu V, Ganić E, Hartjes S, Babić O, Curran R (2019) Air traffic assignment based on daily population mobility to reduce aircraft noise effects and fuel consumption. Transp Res Part D: Transp Environ 72:127-147. https://doi.org/10.1016/j.trd.2019.04.007

20. Barbosa H et al (2018) Human mobility: models and applications. Phys Rep 734:1-74. https:// doi.org/10.1016/j.physrep.2018.01.001

21. Girardin F, Calabrese F, Fiore FD, Ratti C, Blat J (2008) Digital footprinting: uncovering tourists with user-generated content. IEEE Pervasive Comput 7(4):36-43. https://doi.org/10. 1109/MPRV.2008.71 
22. Castiglione J, Bradley M, Gliebe J (2014) Activity-based travel demand models: a primer. Transportation Research Board, Washington, D.C.

23. Ganić E, van Oosten N, Meliveo L, Jeram S, Louf T, Ramasco JJ (2020) Dynamic noise maps for Ljubljana airport. In: 10th SESAR innovation days

24. Statistical Office of the Republic of Slovenia (2019) In: Methodological explanation daily passenger mobility. Ljubljana, Slovenia

25. European Commission (2002) Position paper on dose response relationships between transportation noise and annoyance

Open Access This chapter is licensed under the terms of the Creative Commons Attribution 4.0 International License (http://creativecommons.org/licenses/by/4.0/), which permits use, sharing, adaptation, distribution and reproduction in any medium or format, as long as you give appropriate credit to the original author(s) and the source, provide a link to the Creative Commons license and indicate if changes were made.

The images or other third party material in this chapter are included in the chapter's Creative Commons license, unless indicated otherwise in a credit line to the material. If material is not included in the chapter's Creative Commons license and your intended use is not permitted by statutory regulation or exceeds the permitted use, you will need to obtain permission directly from the copyright holder. 